\title{
DESARROLLO DE UN ALGORITMO DE SOLUCIÓN DIRECTA PARA EL CÁLCULO DE DISTRIBUCIONES ÓPTIMAS DE CULTIVOS BAJO RIEGO DEFICITARIO CONTROLADO
}

\author{
López-Mata, $E .{ }^{1}(P)$, Orengo-Valverde, J.J. ${ }^{2}$, Tarjuelo, J.M. ${ }^{1}$, Martínez-Romero, $A .{ }^{1} y$ \\ Domínguez, A. $^{1}$
}

\begin{abstract}
${ }^{1}$ Investigador, Centro Regional de Estudios del Agua (CREA), Universidad de Castilla-La Mancha, Ctra. de Las Peñas, km 3.2 , 02071 Albacete (Spain), Eulogio.Lopez@uclm.es; Jose.Tarjuelo@uclm.es; Angel.MRomero@uclm.es; Alfonso.Dominguez@uclm.es

${ }^{2}$ Profesor, Departamento de Matemáticas, Área de Matemática Aplicada, Universidad de Castilla-La Mancha, Campus Universitario, s/n,02071, Albacete, (Spain), Jose.Orengo@uclm.es
\end{abstract}

\section{Resumen}

La adecuada gestión de un recurso natural cada vez más escaso, como es el agua, implica maximizar la eficiencia en su uso. Desde el punto de vista de una explotación agraria, es de la máxima importancia encontrar la distribución óptima de cultivos que maximice el margen bruto obtenido con el agua de riego disponible. Actualmente, los métodos de optimización disponibles para resolver este problema no lineal, recurren a métodos de optimización heurísticos de propósito general mucho más lentos y menos eficientes de lo que sería un algoritmo de optimización de solución directa, donde se conocen los mecanismos involucrados y las sinergias existentes entre los cultivos para la obtención de la solución óptima del problema. El objetivo del presente trabajo es desarrollar un algoritmo de solución directa capaz de determinar la distribución óptima de cultivos que sea compatible con el modelo MOPECO (Modelo de Optimización Económica del agua de riego). La solución óptima se consigue con sólo uno o dos cultivos, pero esta solución no es la más adecuada desde el punto de vista agronómico (p.e. rotación de cultivos, PAC, etc.), por lo que ha sido necesario adaptar el algoritmo desarrollado para manejar este tipo de situaciones. Para una hipotética explotación de 100 ha, considerando 10 cultivos diferentes y 11 escenarios de volumen de agua total disponible, se han comparado los resultados del algoritmo desarrollado con las soluciones ofrecidas por el software de optimización LINGO y los algoritmos genéticos. El algoritmo desarrollado consigue márgenes brutos un $0,5 \%$ inferiores a los de LINGO, y un 1,1\% mayores que los algoritmos genéticos, reduciendo el tiempo de cálculo entre 50-100 y 2000 veces, respectivamente.

\section{1- Introducción}

El agua es un recurso escaso que debe ser gestionado de forma eficiente. Desde mediados del siglo XX se han desarrollado modelos complejos como Aquacrop, ISAREG, CROPSYS o WOFOST, que permiten simular el comportamiento de los cultivos bajo un amplio rango de condicionantes. MOPECO (Ortega et al., 2004) ha sido concebido para la optimización del margen bruto (MB) de las explotaciones de riego, en especial en zonas con escasos recursos hídricos y/o con altos costes de cultivo. Bajo condiciones reales, el sector productivo requiere de una herramienta que les asesore sobre cual es la superficie y el volumen de agua de riego, normalmente deficitario, que debe asignar a cada cultivo para maximizar la rentabilidad. Los datos requeridos para realizar esta tarea de optimización son las relaciones entre el margen bruto y la lámina de riego. Estas relaciones no son lineales, 
por lo que se deben de utilizar técnicas de optimización no lineal durante el proceso de optimización.

En la actualidad MOPECO utiliza los algoritmos genéticos para resolver este problema de optimización. Esta metodología presenta dos problemas. Por una parte, el resultado obtenido no es el óptimo absoluto. Y, por otro lado, el tiempo de cálculo requerido es muy elevado, lo que limita la posibilidad de generar una versión on-line del modelo para su utilización por los regantes y gestores de amplias zonas regables con un elevado número de usuarios.

El objetivo principal del presente trabajo es desarrollar una metodología capaz de determinar la distribución de cultivos que maximiza el margen bruto de una explotación, conocida la superficie regable y la cantidad de agua disponible, así como las funciones margen bruto vs. lámina de riego de cada cultivo, con bajos requerimientos de tiempo de cálculo. Los objetivos específicos son: i) Desarrollar un algoritmo de solución directa para el cálculo de distribuciones óptimas de cultivos bajo riego compatible con MOPECO; ii) Mejorar la eficiencia y velocidad en la resolución de problemas de distribución óptimas de cultivos respecto a los algoritmos genéticos; iii) Analizar los mecanismos y sinergias entre los cultivos para obtener la solución óptima.

\section{2- Materiales y métodos}

\section{1- Formulación del problema}

El problema de optimización de la distribución de cultivos en una explotación agraria bajo condiciones de estrés hídrico se puede formular de la siguiente manera:

A partir de una parcela de superficie $\left(S_{\text {total }}\right)$, una dotación de volumen de agua de riego $\left(\mathrm{V}_{\text {total }}\right)$, unos cultivos de los que se conocen sus funciones margen bruto frente a la lámina de riego aplicada, se desean encontrar los valores de superficie $\left(S_{i}\right)$ y volumen de agua de riego ( $\mathrm{Vi})$ que maximicen el margen bruto de la explotación ( $\left.\mathrm{MB}_{\text {total }}\right)$, expresión (1). Siendo: $\mathrm{n}$ el número total de cultivos a tener en cuenta en el problema y $\mathrm{f}$ la función que representa el margen bruto obtenido con respecto a la lámina aplicada (función MB/lam). La ecuación (1) está sujeta a las restricciones (2), (3), (4), (5) y (6):

$$
\begin{aligned}
\mathrm{MB}_{\text {total }}=\sum_{\mathrm{i}=1}^{\mathrm{n}} \mathrm{S}_{\mathrm{i}} \cdot \mathrm{f}_{\mathrm{i}}\left(\frac{\mathrm{V}_{\mathrm{i}}}{\mathrm{S}_{\mathrm{i}}}\right) \\
\mathrm{S}_{\text {total }} \geq \sum_{\mathrm{i}=1}^{\mathrm{n}} \mathrm{S}_{\mathrm{i}} \\
\mathrm{V}_{\text {total }} \geq \sum_{\mathrm{i}=1}^{\mathrm{n}} \mathrm{V}_{\mathrm{i}} \\
\mathrm{S}_{\mathrm{i}} \geq 0 \\
\mathrm{~V}_{\mathrm{i}} \geq 0 \\
\operatorname{lam}_{\mathrm{i}} \leq \operatorname{lam}_{\text {imax }}
\end{aligned}
$$

\section{2- "Funciones margen bruto / lámina"}

Las funciones MB/Lam dependen de las condiciones particulares de cada temporada (climatología, precio de venta de la cosecha, costes de cultivo, etc.). Siendo las mismas diferentes para cada año. Además, otros factores, como la aplicación de un adecuado 
calendario de riegos durante la etapa de crecimiento lo que afecta al MB final para una misma cantidad de agua aplicada al cultivo. López-Mata et al. (2010) simuló 146410 calendarios de riego del cultivo maíz modificando el déficit hídrico y la eficiencia del sistema de riego de forma aleatoria. Una línea de puntos denominada "Frontera de Pareto" (Darshana et al., 2011) representa el máximo MB posible por cada lámina suministrada al cultivo (Figura 1). En ésta línea, es imposible mejorar la variable MB sin perjudicar a la variable lámina de riego.

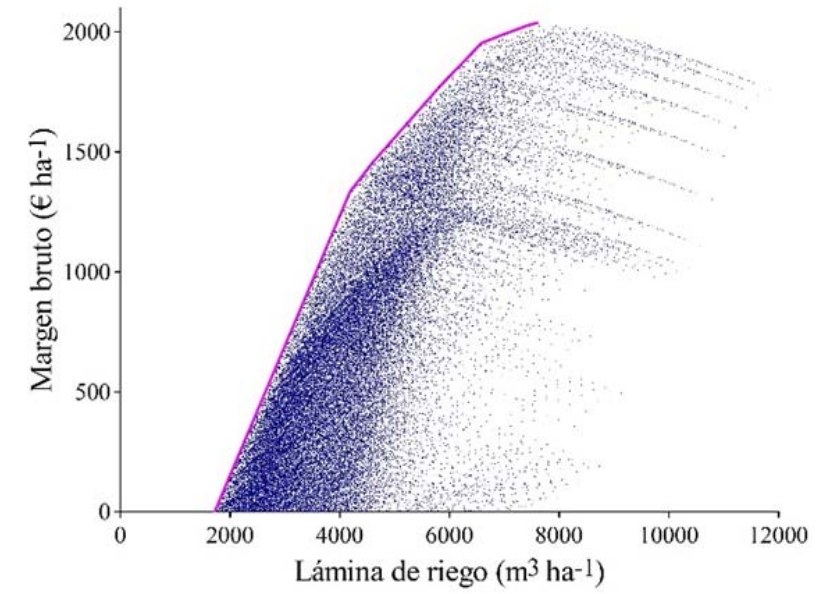

a

Figura. 1. a) Simulación de 146.410 calendarios de riego para el cultivo maíz. b) Algoritmo de recubrimiento.

Es inaceptable, en términos de tiempo de cálculo, simular una gran cantidad de calendarios de riego para obtener la frontera de Pareto del cultivo. Por ese motivo, MOPECO utiliza la metodología de riego deficitario controlado (ORDI) para determinar el calendario de riegos óptimo para una determinada cantidad de agua de riego (Domínguez et al., 2012a). La serie de datos climáticos utilizada por MOPECO es el año meteorológico típico (TMY) de la zona (Domínguez et al., 2013), consiguiendo de ésta forma una única función (MB/lam) por cultivo, teniendo en cuenta el valor medio de ciertos parámetros económicos.

\section{3- Algoritmo de optimización}

Se ha determinado matemáticamente que el óptimo margen bruto de la ecuación (1) con un solo cultivo con una función (MB/lam) positiva, creciente y cóncava (Figura 2a), denominada ideal en el presente trabajo, se obtiene cuando todo el volumen de agua es aplicado a toda la superficie disponible de la parcela $\left(\operatorname{lam}_{\mathrm{opt}}=\mathrm{V}_{\text {total }} / \mathrm{S}_{\text {total }}\right)$. Si se dispusiera de un volumen de agua superior a $S_{\text {total }} \cdot \operatorname{lam}_{M B m a x}$, la solución óptima sería $S=S_{\text {total }}$ y $\mathrm{V}=\mathrm{S}_{\text {total }} \cdot \operatorname{lam}_{\mathrm{MBmax}}$, regando con lam $=$ lam $_{\mathrm{MBmax}}$.

En los siguientes apartados, se desarrollarán una serie de transformaciones factibles donde se convertirá un problema con cualquier número de cultivos, con cualquier forma MB/lam en una única función MB/lam ideal, con una respuesta de margen bruto mejor y cuyo óptimo ya se ha determinado anteriormente.

\subsection{Consideraciones sobre el reparto de agua}

Una misma dotación de agua se puede aplicar uniformemente a la totalidad de la parcela o se puede dividir en dos zonas de riego con diferentes láminas de agua aplicada, siendo el margen bruto medio por hectárea diferente. 
La figura (2b) muestra 3 funciones MB/lam que pasan todas ellas por dos puntos $\mathrm{P}_{1}$ y

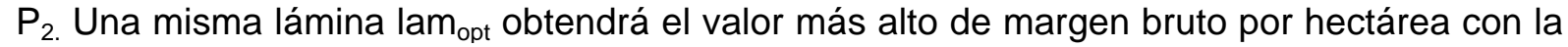
función cóncava. Con la función convexa, la parcela puede ser dividida en dos superficies de riego diferentes con el mismo cultivo, una regada con la lámina $\operatorname{lam}_{1}$ (punto $\mathrm{P}_{1}$ ) y la otra regada con la lámina $\operatorname{lam}_{2}$ (punto $\mathrm{P}_{2}$ ) elegidas de tal forma que la lámina media coincida con

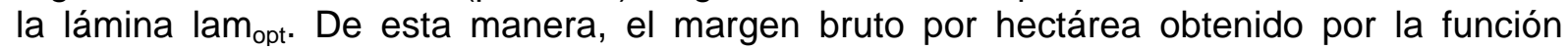
convexa es igual al obtenido por la función lineal. Obteniendo las siguientes expresiones

$$
\begin{gathered}
\mathrm{S}_{2}=\mathrm{S}_{\text {total }} \cdot\left(\frac{\operatorname{lam}_{\mathrm{opt}}-\mathrm{lam}_{1}}{\operatorname{lam}_{2}-\operatorname{lam}_{1}}\right) \\
\mathrm{V}_{2}=\mathrm{S}_{2} \cdot \operatorname{lam}_{2} \\
\mathrm{~V}_{1}=\mathrm{V}_{\text {total }}-\mathrm{V}_{2} \\
\mathrm{~S}_{1}=\mathrm{S}_{\text {total }}-\mathrm{S}_{2}
\end{gathered}
$$

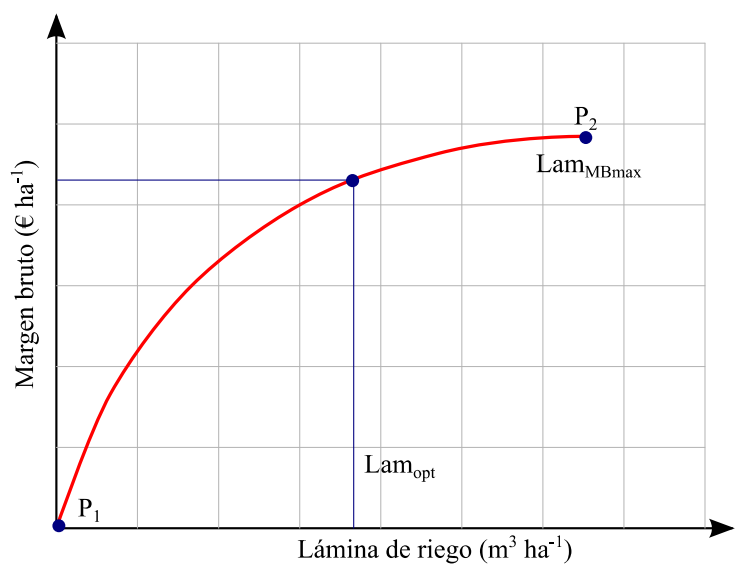

a

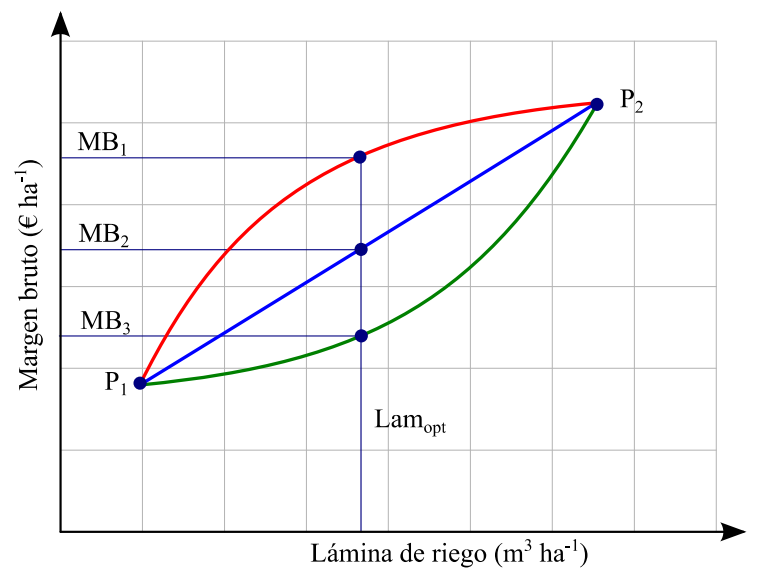

b

Figura 2. a) Función MB/lam “ideal”; b) Comparaciones entre funciones MB/lam cóncava, lineal y convexa que pasen por los mismos dos puntos.

\subsection{Transformación de funciones MB/Lam no cóncavas ni crecientes}

Si la función MB/Lam no es creciente y/o cóncava, la función se puede transformar detectando estas partes de la misma (p.e., la parte comprendida entre los puntos $P_{1}$ y $P_{2}$ de la figura 3a) y trazando una línea que una ambos puntos (Apartado 2.4). Esta línea será utilizada como nueva función $\mathrm{MB} / \mathrm{lam}$ entre los puntos $\mathrm{P}_{1}$ y $\mathrm{P}_{2}$ y está definida por las ecuaciones (7), (8), (9), y (10).

\subsection{Transformación de funciones MB/lam no siempre positivas}

Estas funciones pueden ser transformada de la misma forma que en la transformación anterior añadiendo un cultivo virtual con una función MB/lam constituida por solo un punto (lam $=0 \mathrm{~m}^{3} \mathrm{ha}^{-1}$ y $\mathrm{MB}=0 € \mathrm{ha}^{-1}$ ) (Figura $3 b$ ).

\subsection{Transformación de $\mathbf{n}$ funciones MB/lam en una sola función}

Para el caso de dos cultivos (Figura 4a), el cultivo 1 responde mejor con láminas de riego bajas, mientras que el cultivo 2 responde mejor a láminas altas. Por lo tanto, la nueva función MB/lam estará formada, desde la lámina lam=0 hasta lam $_{1}$ por la función MB/lam del 
cultivo 1, entre $\operatorname{lam}_{1}$ y $\operatorname{lam}_{2}$ por una línea tangente a las funciones de ambos cultivos, y desde lam $_{2}$ la función MB/lam del cultivo 2. El procedimiento para encontrar el óptimo para cualquier número de cultivos es simplemente una generalización del procedimiento para dos cultivos (Figura 4b).

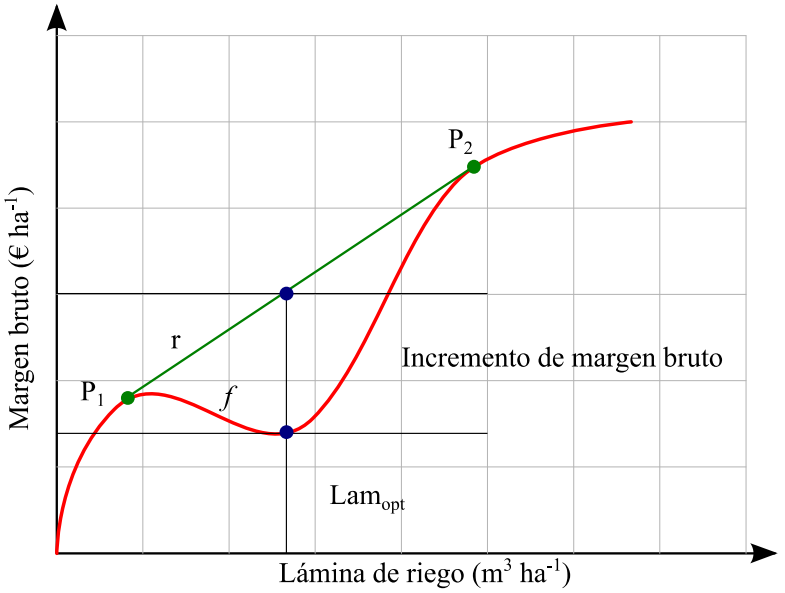

a

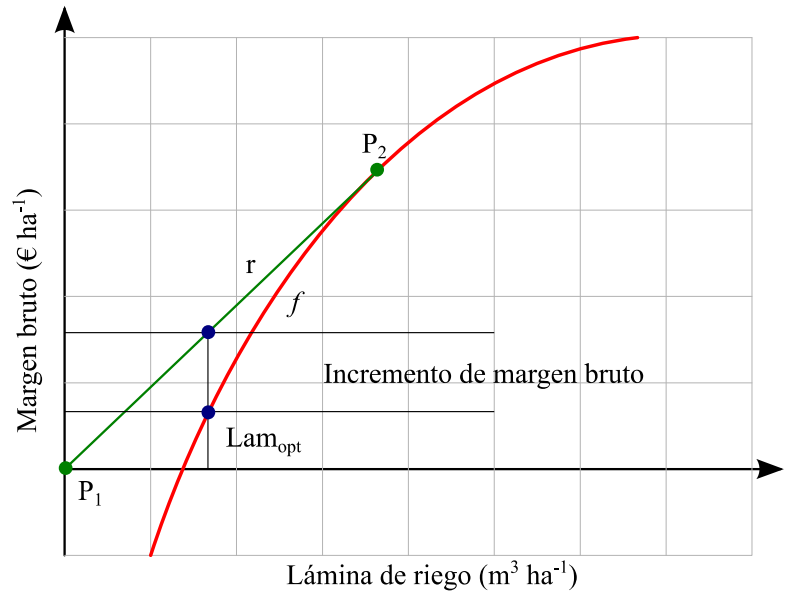

b

Figura 3. a) Transformación de una función MB/Lam no cóncava y no creciente; b) Transformación de una función MB/lam no siempre positiva.

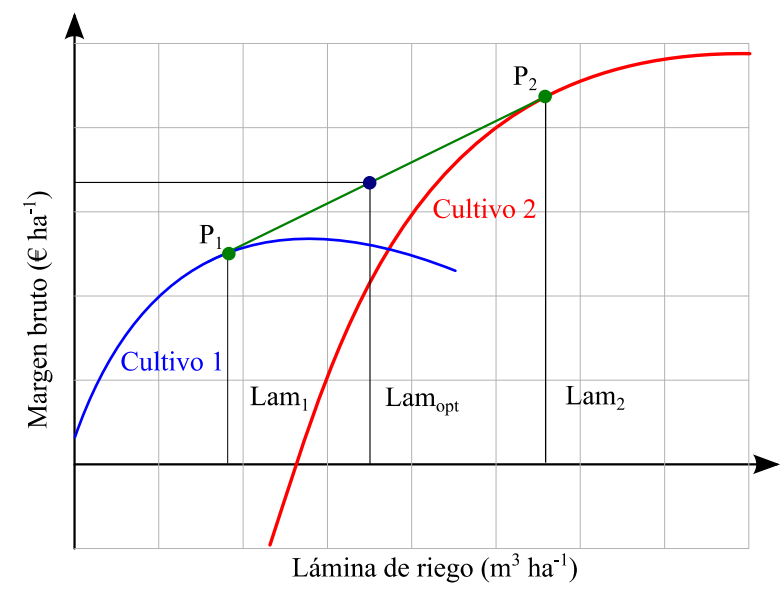

a

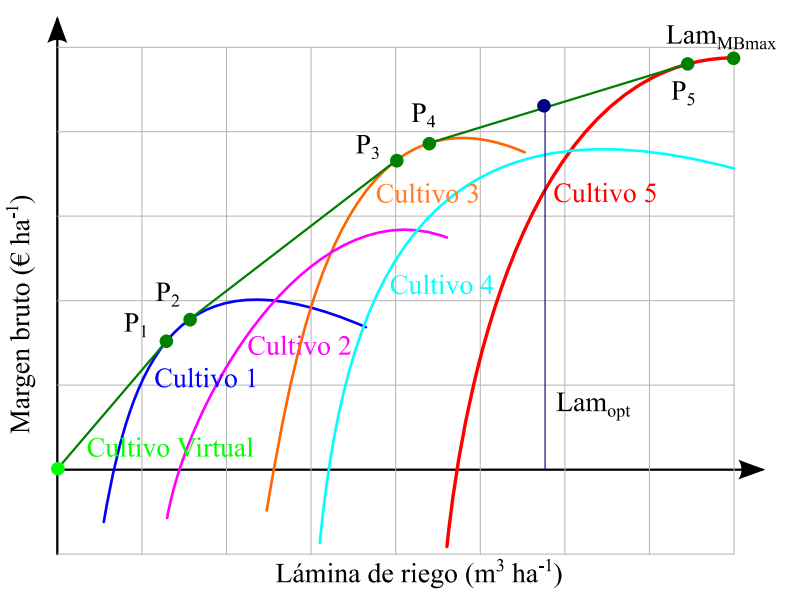

$\mathrm{b}$

Figura 4. a) Transformación de las curvas MB/lam de dos cultivos en una sola curva MB/lam; b) Transformación de las funciones MB/lam de 5 cultivos en una sola función.

\subsection{Algoritmo de recubrimiento}

Para seleccionar únicamente los puntos pertenecientes a la "frontera de Pareto" de los cultivos y aplicar todas las transformaciones explicadas se ha desarrollado un algoritmo de recubrimiento, que lo procesa todo a la vez. El algoritmo ha sido diseñado para trabajar con listas de puntos.

Como primer punto de referencia se elige el primer punto de la lista ordenada de puntos, a continuación, se calculan las pendientes de los puntos que lo siguen con respecto al punto de referencia, eligiendo como segundo punto de referencia el punto de mayor pendiente positiva. Los puntos entre ambos puntos de referencia son eliminados. Este proceso es repetido sucesivamente hasta llegar al final de la lista, obteniendo una función cóncava (Figura 1b). 


\subsection{El proceso de optimización}

El proceso de optimización consta de dos procesos diferentes. El primer proceso determina la "Frontera de Pareto" de cada uno de los cultivos, y el segundo proceso determina la distribución óptima en términos de superficie y el volumen de agua para el conjunto de los cultivos considerados, siguiendo la metodología descrita en los puntos anteriores.

\subsection{Adaptación del algoritmo a la gestión real de explotaciones agrarias.}

Se ha demostrado, hasta este punto, que la solución óptima del problema de distribución de cultivos es o bien uno o dos cultivos. En la gestión de explotaciones agrarias reales, hay numerosos argumentos en favor de limitar la superficie máxima de un cultivo individual y adoptar la rotación de cultivos. Consecuentemente, la implementación de ésta metodología en el modelo MOPECO requiere que los usuarios puedan limitar la superficie máxima de cada cultivo. La manera de resolver éste problema es la siguiente:

Paso 1. Realizar la optimización siguiendo los pasos descritos en el apartado 2.9.

Paso 2. Si la superficie asignada para cualquiera de los cultivos de la solución obtenida es mayor a la máxima permitida por el usuario, el exceso de superficie y su correspondiente volumen de agua serán utilizados en una nueva optimización en la cual las funciones MB/lam de los cultivos que han alcanzado la superficie máxima son eliminadas. Este proceso debe ser repetido hasta que sea posible.

Paso 3. Es posible que una cierta cantidad de agua quede disponible al final de la última optimización. Éste volumen de agua es asignado al cultivo con el mayor incremento de margen bruto por unidad de agua suministrada (la función MB/lam con mayor pendiente positiva para la lámina determinada), pudiendo incluso ser asignado a varios cultivos.

\subsection{Evaluación de la metodología desarrollada.}

Se han utilizado diez cultivos ficticios para evaluar la distribución de cultivos que maximice el beneficio de una explotación agraria con el algoritmo desarrollado bajo dos escenarios: sin restricción de superficie a los cultivos (sin rotación), y siguiendo la metodología descrita en el apartado 2.10 considerando restricciones de superficie en los cultivos (rotación). En éste último caso, se ha considerado que la máxima superficie para cada cultivo sea $1 / 3$ de la superficie total de la explotación. Las funciones MB/lam utilizadas son funciones polinómicas, tal y como sugieren diferentes autores (English et al., 2002; Domínguez et al., 2012b,c) (Figura 5). Para este ejemplo, la superficie de riego total ha sido de 100 ha, y se han considerado 11 volúmenes de agua de riego. Los resultados con limitación de superficie en cultivos has sido comparados con los obtenidos por los algoritmos genéticos y el software de optimización LINGO (LINDO, 2015).

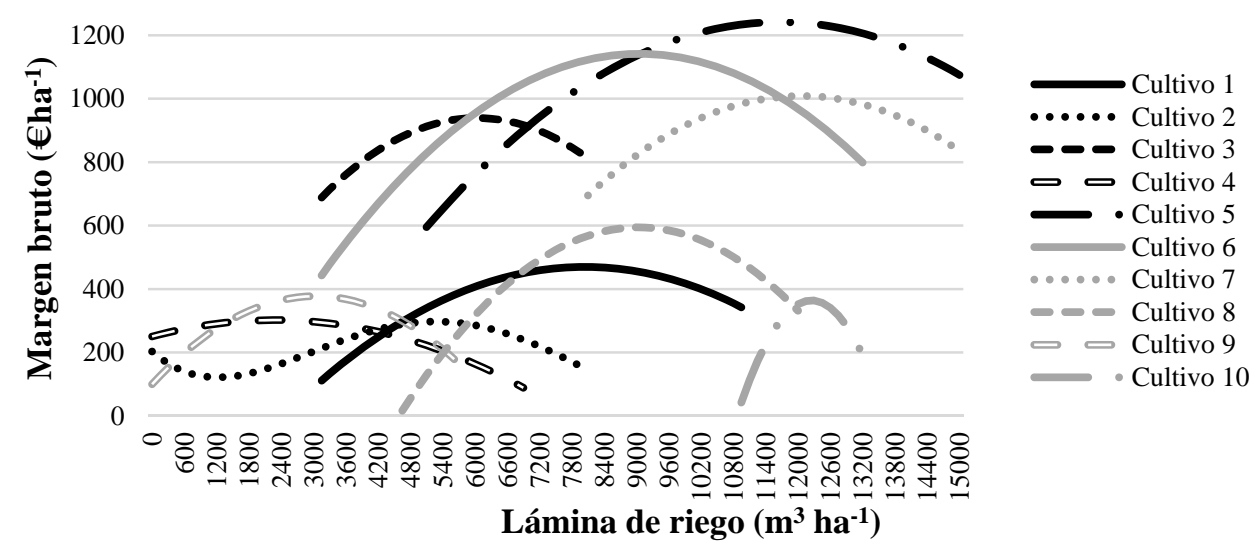


Figura 5. Funciones "MB/lam" de 10 cultivos ficticios.

\section{Resultados y discusión}

La principal diferencia entre las metodologías de optimización ha sido el tiempo de cálculo (Tabla 1). El algoritmo propuesto (AP) ha sido capaz de obtener los resultados 2000 veces más rápido que los algoritmos genéticos (AG), y entre 10-100 veces respecto a LINGO. Éste hecho es particularmente relevante para el desarrollo futuro de una versión online del modelo MOPECO para su utilización por agricultores. El margen bruto alcanzado por el " AP (rotación)" es siempre más bajo que el margen bruto alcanzado por el "AP (sin rotación)" (disminución media igual a 7,1\%), mientras que los requisitos de tiempo de cálculo fueron similares (Tabla 1).

Tabla 1. Resumen de los resultados de la comparación de los diferentes algoritmos.

\begin{tabular}{|c|c|c|c|c|c|c|c|c|}
\hline & \multicolumn{2}{|c|}{ AP (sin rotación) } & \multicolumn{2}{|c|}{ AP (rotación) } & \multicolumn{2}{|c|}{ AG (rotación) } & \multicolumn{2}{|c|}{ LINGO (rotación) } \\
\hline $\begin{array}{l}\text { Agua } \\
\left(\mathrm{m}^{3}\right)\end{array}$ & $\begin{array}{c}\text { MB } \\
(€) \\
\end{array}$ & $\begin{array}{c}t \\
(s)\end{array}$ & $\begin{array}{l}\text { MB } \\
(€)\end{array}$ & $\begin{array}{c}t \\
(s)\end{array}$ & $\begin{array}{l}M B \\
(€)\end{array}$ & $\begin{array}{l}t \\
(s)\end{array}$ & $\begin{array}{c}\text { MB } \\
(\boldsymbol{E})\end{array}$ & $\begin{array}{c}t \\
(s)\end{array}$ \\
\hline 0 & 25000,0 & 0,02035 & 18428,4 & 0,02165 & 18428,4 & 26,21 & 18428,4 & 1 \\
\hline 130000 & 43436,0 & 0,02044 & 41703,4 & 0,02041 & 40698,8 & 41,56 & 41819,7 & 1 \\
\hline 260000 & 61871,9 & 0,02048 & 58050,5 & 0,02015 & 57903,9 & 52,72 & 58105,6 & 2 \\
\hline 390000 & 80173,8 & 0,02036 & 72909,2 & 0,02 & 7200 & 44,41 & 73274,9 & 1 \\
\hline 520000 & 92287,3 & 0,02045 & 85939,2 & 0,02025 & 86106,5 & 46,53 & 86145,1 & 2 \\
\hline 650000 & 102002,4 & 0,02066 & 98331,0 & 0,02052 & 97142,4 & 46,53 & 99009,7 & 1 \\
\hline 780000 & 110976,4 & 0,02047 & 107491,8 & 0,02020 & 104185,6 & 40,78 & 108224,0 & 2 \\
\hline 910000 & 116422,7 & 0,02024 & 110264,0 & & 109601,1 & 43,26 & 111118,0 & 2 \\
\hline 1040000 & 121752,5 & 0,02026 & 112204,3 & 0,02018 & 110926,9 & 51,11 & 112632,4 & 2 \\
\hline 1170000 & 124165,0 & 0,02014 & 113054,3 & 0,02029 & 111036,7 & 45,99 & 113059,6 & $<1$ \\
\hline 1300000 & 124165,0 & 0,02029 & 113054,3 & 0,02025 & 111709,9 & 53,07 & 113059,6 & $<1$ \\
\hline
\end{tabular}

Donde: MB: margen bruto; t: tiempo

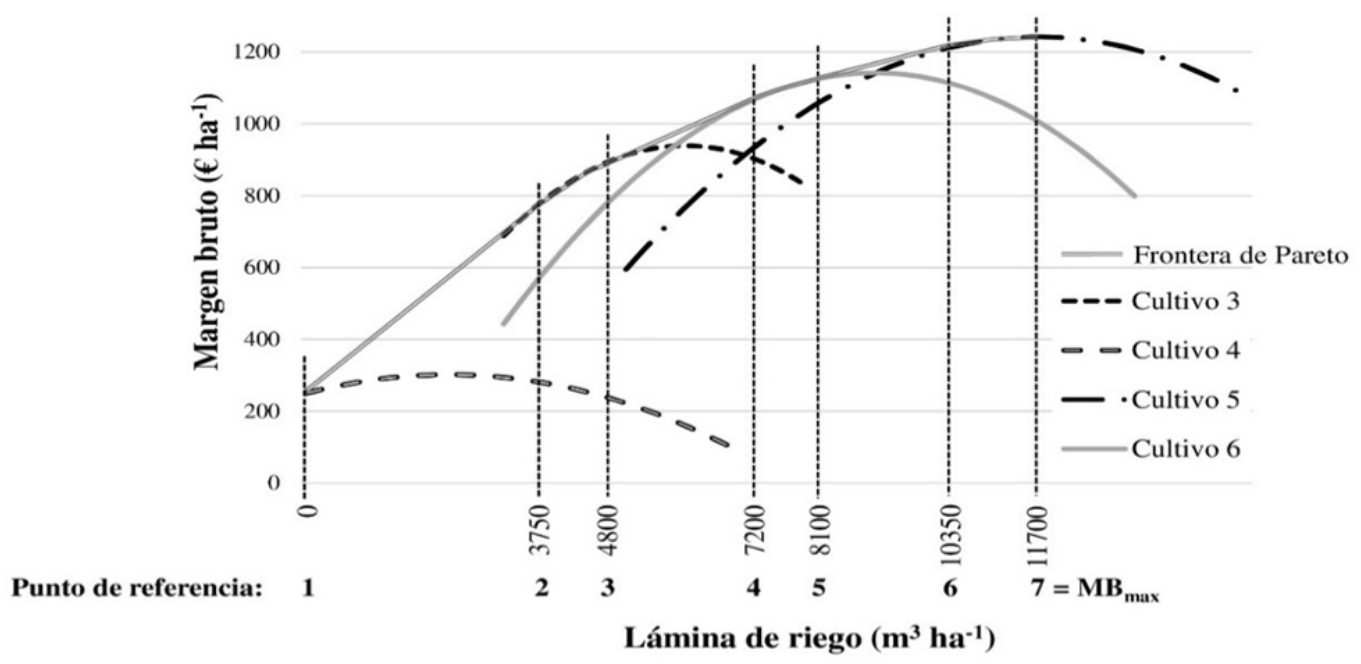

Figura 7. "Frontera de Pareto" y puntos de referencia obtenidos por el algoritmo desarrollado.

Los resultados del algoritmo propuesto con las restricciones de superficie "AP (Rotación)" se han comparado con los generados por "LINGO (Rotación)" y "AG (Rotación)", también con límites de superficie. En términos generales, las distribuciones de cultivos propuestas por las tres metodologías fueron muy similares en términos de cultivos seleccionados en la mayoría de los escenarios, difiriendo ligeramente en los valores de superficie y lámina asignados. Las diferencias en el uso de agua disponible aumentaron con 
la disponibilidad de agua, donde los AG dejaban recursos sin utilizar en cuatro escenarios. En todos los casos, se ha utilizado toda la superficie, mientras que en los dos últimos escenarios se ha dejado agua sin utilizar. LINGO ha sido la metodología que ha obtenido los valores más altos de margen bruto en todos los escenarios, aunque las diferencias entre ellos son pequeñas (inferiores a 3,9\% para los AG y 2,2\% para el AP, siendo la diferencia media del $1,5 \%$ y $0,5 \%$, respectivamente). Es necesario destacar que la adaptación del algoritmo propuesto para la gestión de una explotación con restricciones de superficie de cultivos puede no ser la óptima, lo que permite a los AG y LINGO lograr mejores resultados en algunos escenarios (Tabla 1). Por el contrario, el algoritmo sin restricciones de superficie (sin rotación) siempre obtiene la solución óptima global. En consecuencia, el algoritmo desarrollado genera resultados que son similares a los obtenidos por los otros dos métodos anteriormente probados. AG es la metodología con los más altos requerimientos de tiempo de cálculo.

Otro resultado relevante es que, para cada cultivo, el algoritmo determina láminas de riego inferiores a la lámina de máximo margen bruto (Figura 7), excepto en los dos últimos escenarios debido a una alta disponibilidad de agua de riego. Este hecho pone de manifiesto la importancia del riego deficitario controlado en la gestión económica de las explotaciones de regadío con baja disponibilidad de agua, según lo recomendado por otros autores (English et al., 2002; Ortega et al., 2004; Georgiou and Papamichail, 2008).

La combinación del algoritmo de optimización propuesto con ORDI en el modelo MOPECO puede ayudar a los agricultores a mejorar la eficiencia del uso del agua y la rentabilidad de sus explotaciones. Este modelo también puede ayudar a los mismos a determinar distribución de cultivos más rentable y a aplicar estrategias de riego deficitario controlado a gran escala.

\section{Conclusiones y recomendaciones}

El algoritmo propuesto determina la superficie y la lámina de riego para un conjunto de cultivos seleccionados que maximiza el margen bruto de la explotación. La determinación precisa de estas funciones es crucial para obtener resultados fiables en condiciones reales.

En comparación con LINGO y con los algoritmos genéticos, el tiempo de cálculo y los recursos computacionales requeridos por el algoritmo desarrollado para la obtención de los resultados de los escenarios analizados son entre 50-100 y alrededor de 2000 menor, respectivamente.

Si no se limita la superficie máxima cultivable para cada uno de los cultivos, la solución óptima al problema de optimización es uno o dos cultivos. Tres o más cultivos nunca obtendrán el máximo margen bruto absoluto. Sin embargo, bajo condiciones de manejo para explotaciones de regadío, hay muchos argumentos a favor de la limitación de la superficie máxima ocupada por un cultivo individual.

La adaptación de la metodología propuesta para resolver esta limitación obtiene resultados adecuados, consiguiendo unos márgenes brutos alrededor del 0,5\% inferior a LINGO, y un 1,1 \% más alto que los algoritmos genéticos para los casos de este estudio.

La mayor parte de los resultados obtenidos por el algoritmo proponen el manejo de los cultivos en condiciones de riego deficitario.

La velocidad de cálculo del algoritmo de optimización propuesto permite utilizarlo como parte de algoritmos de optimización más complejos que tengan en cuenta otros factores. Aún más importante que este hecho en la búsqueda de la distribución óptima de cultivos es la de sacar a la luz los mecanismos involucrados y cuáles son las sinergias entre los diferentes cultivos para la consecución de la solución óptima, permitiendo su conocimiento a los agricultores y técnicos desarrollar mejores estrategias de riego. En este sentido, el funcionamiento del algoritmo es fácil de entender y replicar, siendo posible hacerlo de forma manual. Esta metodología nace de la necesidad de optimización de la distribución de cultivos, pero es razonable pensar que puede ser aplicada en otros campos.

\section{Agradecimientos}


En este trabajo se ha desarrollado en el marco de dos proyectos europeos financiados por EC: FLOW-AID "Farm Level Optimal Water Management: Assistant for Irrigation under Deficit" No. 036958 (GOCE), y DeSURVEY "A Surveillance System for Assessing and Monitoring of Desertification" (SUSTDEV-CT-2004-003950-2).

\section{Bibliografía}

Darshana, Pandey, A., Ostrowski, M., Pandey, R.P., 2011. Simulation and optimization for irrigation and crop planning. Irrigation and Drainage 61, 178-188.

Domínguez, A., Martínez-Romero, A., Leite, K.N., Tarjuelo, J.M., de Juan, J.A., López-Urrea, R., 2013. Combination of typical meteorological year with regulated deficit irrigation to improve the profitability of garlic growing in central Spain. Agricultural Water Management 130, 154-167.

Domínguez, A., de Juan, J.A., Tarjuelo, J.M., Martinez, R.S., Martinez-Romero, A., 2012a. Determination of optimal regulated deficit irrigation strategies for maize in a semi-arid environment. Agricultural Water Management 110, 67-77.

Domínguez, A., Jimenez, M., Tarjuelo, J.M., de Juan, J.A., Martinez-Romero, A., Leite, K.N., 2012b. Simulation of onion crop behavior under optimized regulated deficit irrigation using MOPECO model in a semi-arid environment. Agricultural Water Management $113,64-75$.

Domínguez, A., Martinez, R.S., de Juan, J.A., Martinez-Romero, A., Tarjuelo, J.M., 2012c. Simulation of maize crop behavior under deficit irrigation using MOPECO model in a semi-arid environment. Agricultural Water Management 107, 42-53.

English, M.J., Solomon, K.H., Hoffman, G.J., 2002. A paradigm shift in irrigation management. Journal of Irrigation and Drainage Engineering 128, 267-277.

Georgiou, P.E., Papamichail, D.M., 2008. Optimization model of an irrigation reservoir for water allocation and crop planning under various weather conditions. Irrigation Science 26, 487-504.

LINDO, 2015. LINGO. The modeling language and optimizer. User's manual. LINDO Systems Inc., Chicago, USA.

López-Mata, E., Tarjuelo, J.M., de Juan, J.A., Ballesteros, R., Domínguez, A., 2010. Effect of irrigation uniformity on the profitability of crops. Agricultural Water Management 98, 190-198.

Ortega, J.F., de Juan, J.A., Tarjuelo, J.M., López-Mata, E., 2004. MOPECO: an economic optimization model for irrigation water management. Irrigation Science 23, 61-75. 